\title{
Hurricanes: lessons from Charley and the gang
}

Background and epidemiology: Immediately after Florida experienced 4 major hurricanes (Charley, Frances, Ivan and Jeanne) in quick succession during August and September of 2004, the Florida Department of Health deployed the Behavioral Risk Factor Surveillance System to assess the effect of the hurricanes on residents' health. ${ }^{1}$ In October nearly 2000 respondents answered 30 questions on topics such as their level of preparedness, physical injuries experienced, use of portable generators and work absences. ${ }^{1}$

This telephone survey found that before the hurricanes, nearly half of Floridians (48.7\%) had no evacuation plan. Rather surprisingly, incidences of health consequences (physical injuries, lost workdays and loss of access to necessary medical treatment, including equipment and supplies) among people living in Florida counties lying outside the direct path of any of the 4 hurricanes were similar to those of residents of counties that had

\section{Box 1: Hurricanes in Canada}

Hurricanes are tropical storms in which winds of $120 \mathrm{~km} / \mathrm{h}$ or more revolve around a centre of low atmospheric pressure called the eye.

They are unusual in Canada, occurring occasionally in the east (from the eastern Lake Superior area to the Atlantic provinces) and much more rarely along the west coast.

Hurricanes form over warm ocean waters, generally between latitudes $5^{\circ}$ and $25^{\circ}$ north or south of the equator.

Most of those affecting North America originate off the west coast of Africa. They tend to travel northwest and then northeast, dissipating their energy over cooler waters and frictional land. Thus, hurricanes rarely strike Canada in full fury. been directly affected (Table 1). These data imply that people living in the predicted path of a hurricane and those living in adjacent areas are about equally vulnerable. Orders for evacuation should therefore consider and possibly extend to nearby areas, especially when particular hazards, such as a potential for flooding, exist.

Portable generators were used after electrical power outages in about 1 in 6 homes, resulting in at least 173 cases of carbon monoxide $(\mathrm{CO})$ poisoning (largely caused by running a generator indoors). ${ }^{\prime}$ This result particularly resonates with Canadian health officials, who in 1998 witnessed some 2 dozen cases of CO poisoning in central Canada in the aftermath of the notorious ice storm. ${ }^{2}$

\section{Preparedness and prevention:} Emergency preparedness requires that both the content and process of disaster planning be addressed. Several Ontario jurisdictions have adopted the incident management system (IMS) protocol (see also www.fema .gov/nims). IMS calls for the appointment of an incident commander and officers to cover the essential duties of administration and financial control, information, liaison, logistics, operations, planning and safety. Local physicians (both public health and clinical) and key stakeholders contribute greatly to disaster planning and response.

Community members with chronic conditions such as diabetes, cardiovascular disease and asthma should be warned of storms well in advance. Because they have special needs such as access to medications, therapy or specialized equipment (e.g., dialysis or oxygen), they need to have contingency plans in place to ensure access to vital care.

Portable generators are becoming more widely available for private use during power outages. The public needs to be educated about their safe operation and the hazards of CO.

Not all communities face the same hazards (Box 1), ${ }^{3}$ but all share a random potential for an idiosyncratic environmental disaster. Planning is most effective

Table 1: Percentage of Florida respondents reporting consequences from hurricanes Charley, Frances, Ivan or Jeanne, 2004

\begin{tabular}{lcc} 
Consequence & $\begin{array}{c}\text { In direct } \\
\text { path }\end{array}$ & $\begin{array}{c}\text { Not directly } \\
\text { in path }\end{array}$ \\
\hline Missed work or lost a job & 53 & 56 \\
Experienced physical injury & 5 & 4 \\
$\begin{array}{lc}\text { Used electrical generator } \\
\text { inside residence }\end{array}$ & 2 & 9 \\
Biggest concern & & \\
$\quad$ Quality of drinking water & 52 & 50 \\
$\quad$ Sewage disposal & 15 & 11 \\
$\quad$ Solid waste problems & 10 & 9 \\
$\quad$ Food protection, storage & 11 & 13 \\
$\quad$ Mosquito control & 5 & 1 \\
\hline
\end{tabular}

when based on the disaster scenarios most likely to occur locally. To ascertain this, local hazards such as flood zones and other potential or actual chemical, biological, physical or environmental circumstances must be inventoried. A transparent, participatory community planning session can then be arranged that takes into account the individuals and institutions likely to be involved.

Erica Weir

CMAF

\section{References}

1. US Centers for Disease Control and Prevention. Epidemiologic assessment of the impact of four hurricanes. Carbon monoxide poisoning from hurricane-associated use of portable generators - Florida, 2004. MMWR Morb Mortal Wkly Rep 2005;54(28): 693-700. Available: www.bt.cdc.gov /disasters/hurricanes/mmwr.asp\#2004 (accessed 2005 Aug 16).

2. Hartling L, Pickett $W$, Brison RJ. The injury experience observed in two emergency departments in Kingston, Ontario during "ice storm '98." Can 7 Public Health 1999;90(2):95-8.

3. Major hurricanes that affected Canada (map). Available: http://atlas.gc .ca/site/english/maps/environment /naturalhazards/majorhurricanes (accessed 2005 Aug 16). 